\title{
Alimentación y nutrición de la población ante situaciones de desastres naturales
}

\section{Feeding and nutrition of the population in natural disaster situations}

\author{
María Matilde Socarrás Suárez; Miriam Bolet Astoviza ${ }^{\mathrm{I}}$ \\ IEspecialista de II Grado en Nutrición. Profesor Auxiliar. Facultad "Calixto García \\ Iñiguez". Hospital Universitario "General Calixto García Iñiguez". La Habana, Cuba.
}

\section{RESUMEN}

Los desastres naturales azotan a Cuba y se deben atenuar sus estragos sobre la salud y la alimentación de la población. El estado de nutrición de la población depende de la disponibilidad, el consumo y la utilización biológica de los alimentos. Los desastres naturales pueden perjudicar el estado nutricional de la población debido a su impacto sobre uno o varios componentes de la cadena alimentaria, que dependerán del tipo, duración y magnitud del desastre, así como de las condiciones de alimentación y nutrición que existían previamente en la zona. Se revisa la información disponible sobre alimentación y nutrición en los desastres y se identifican las medidas de control y prevención para evitar la desnutrición y conseguir la mejor alimentación en las situaciones de emergencia, incluido la alimentación de lactantes y niños pequeños. Se precisan los pasos inmediatos a dar para garantizar la eficacia de un programa de ayuda alimentaria, entre ellos, evaluar las provisiones de alimentos disponibles después del desastre, determinar las necesidades nutricionales de la población afectada, calcular las raciones alimentarias diarias y las necesidades de grandes grupos de población y vigilar el estado de nutrición de la población afectada. En los desastres es necesario mejorar el abastecimiento de alimentos con un mayor aprovechamiento de los productos cosechados y ampliar la cultura nutricional, lo que traería aparejado el incremento de la seguridad alimentaria y la prevención de la desnutrición.

Palabras clave: Desastres naturales, disponibilidad de alimentos, estado nutricional, alimentación, Cuba.

\section{ABSTRACT}


Natural disasters hit Cuba, and so, their effects upon health and feeding of the population should be mitigated. The nutritional condition of the population depends on the availability, consumption and the biological use of food. Natural disasters can damage the nutritional condition of the population due to their impact on one or several food chain components, which will in turn depend on the type, duration and size of the disaster, as well as the existing feeding and nutritional conditions in the area before the disaster. The available information on food and nutrition in disaster situations was reviewed. Also, the control and preventive measures to avoid malnutrition and to provide the best possible food under emergency circumstances, including food for breastfed babies and small children were identified. The immediate steps to assure the food assistance program efficacy were specified. These steps include evaluating the amount of food available after the disaster, determining the nutritional requirements of the affected population, estimating the daily food rations along with the needs of large population groups, and observing the nutritional condition of the affected population. It is necessary to improve the food supply by making a full use of collected products and to expand the nutritional culture, which will lead to increased food safety and to prevention of malnutrition.

Key words: Natural disasters, food availability, nutritional condition, food.

\section{INTRODUCCIÓN}

Los desastres naturales ponen en peligro el bienestar del hombre y su medio ambiente. En Cuba, por ser un país tropical, son frecuentes los fenómenos climáticos como los ciclones. Después de un desastre suele ser necesario evacuar y reasentar a las personas creando concentraciones de población a las que hay que proveer de todos sus alimentos mientras dure su estancia en los albergues. ${ }^{1}$ Esto se realiza en el país desde antes del desastre.

En este trabajo se hace una revisión de la alimentación y nutrición en los desastres naturales, las medidas de control y prevención para evitar enfermedades como la desnutrición.

\section{SALUD, ALIMENTACIÓN Y NUTRICIÓN}

Los riesgos sanitarios posteriores a los desastres no se concretan al mismo tiempo. Las lesiones personales ocurren por lo general en el momento y en el lugar del impacto y requieren atención médica inmediata, mientras que el riesgo de aumento de las enfermedades transmisibles evoluciona más lentamente y adquiere mayor intensidad cuando hay hacinamiento y deterioro de las condiciones higiénicas. ${ }^{2,3}$ La escasez de alimentos en el período posterior al desastre se debe a dos causas: por una parte la destrucción de los depósitos de alimentos en la zona afectada reduce la cantidad de comida disponible, y por la otra, los trastornos en los sistemas de distribución pueden impedir el acceso a los alimentos, incluso cuando no existe una escasez absoluta.

Los desbordamientos de los ríos y las crecidas del mar suelen deteriorar la despensa de los alimentos en los hogares y arruinar los cultivos, interrumpen la 
distribución y provocan penurias locales. ${ }^{1,2}$ En Cuba existe un programa nacional llevado a cabo por la Defensa Civil para enfrentar estas situaciones.

Es frecuente que las necesidades básicas de la población se cubran a partir de fuentes seguras y que no constituyan, en sí, focos de enfermedades infecciosas. Los brotes de gastroenteritis que son las enfermedades que se notifican más frecuentemente en los períodos posteriores a los desastres se relacionan con el hacinamiento, el desplazamiento de la población, la interrupción y contaminación del abastecimiento de agua y de los servicios de saneamiento. ${ }^{1,2}$

El estado de nutrición de la población depende de la disponibilidad, el consumo y la utilización biológica de los alimentos. ${ }^{1}$

Los desastres naturales pueden perjudicar el estado nutricional de la población debido a su impacto sobre uno o varios de los componentes de la cadena alimentaria, que dependerán del tipo, duración y magnitud del desastre, así como de las condiciones de alimentación y nutrición que existían previamente en la zona. ${ }^{1}$

Dentro de la seguridad alimentaria está la distribución equitativa de los ingresos, y la capacidad de obtener los alimentos. Está relacionada con el indicador de mortalidad infantil, mortalidad en menores de 5 años y prevalencia de desnutrición. ${ }^{4,5}$ Según el estado nutricional general de las poblaciones es necesario que el programa alimentario de emergencias, esté destinado fundamentalmente a los grupos más vulnerables.

La finalidad de los programas de nutrición es corregir y prevenir la desnutrición, que la población tenga los medios y conocimientos pertinentes para preparar los alimentos y consumirlos en condiciones seguras y que las personas reciban el apoyo nutricional necesario..$^{1,6-8}$

Para garantizar la eficacia de un programa de ayuda alimentaria, los pasos inmediatos a dar son: evaluar las provisiones de alimentos disponibles después del desastre, determinar las necesidades nutricionales de la población afectada, calcular las raciones alimentarias diarias y las necesidades de la población y vigilar el estado de nutrición de los sujetos afectados. ${ }^{1,4}$

El efecto de los desastres en la utilización biológica de los alimentos, en la absorción intestinal y posterior utilización de nutrientes depende de factores como el impacto del desastre sobre el abastecimiento de agua y los servicios de saneamiento. Esto adquiere una importancia esencial en las infecciones gastrointestinales que afectan la absorción de nutrientes y en otras enfermedades infecciosas que aumentan su demanda. Si inmediatamente después del desastre se produce un aumento de las tasas de desnutrición en los niños pequeños, es más probable que se deba al efecto de las enfermedades gastrointestinales, que a una escasez real de alimentos, lo que hay que tener presente al establecer los mecanismos de vigilancia. ${ }^{1}$

\section{Alimentación de lactantes y niños pequeños}

La lactancia natural es el medio más sano de alimentar a un recién nacido en muchas circunstancias, particularmente en una emergencia, en la que los procedimientos normales de higiene pueden estar alterados y las tasas de infección pueden aumentar. ${ }^{8}$

Cuando se suministran preparaciones para lactantes, se debe tener la seguridad de que la provisión podrá continuar todo el tiempo que el niño lo necesite y que se 
utilice agua hervida para preparar las fórmulas, lavar los biberones y tetes; el lavado correcto de las manos antes de prepararlos y antes de alimentar al bebé.

Los niños pequeños tienen necesidad de alimentos energéticos. Se recomienda que el $30 \%$ del contenido energético de su dieta proceda de lípidos. Cuando los niños de 6 a 24 meses no tienen acceso a la leche materna, los alimentos nutritivos energéticos deben ser suficientes. ${ }^{9}$ (Red Internacional de Grupos Pro Alimentación Infantil IBFAN Colombia. Taller internacional sobre emergencias y desastres "Enfrentando la vulnerabilidad de niños, niñas, mujeres gestantes y madres lactantes". Bogotá, Colombia 28-30 de Noviembre de 2002).

\section{Higiene de los alimentos}

Para mantener la vida en una catástrofe son imprescindibles el oxígeno, el agua y los alimentos. Sin agua se puede sobrevivir solamente por varios días. En todo desastre el abastecimiento de agua potable debe ser revisado desde el sistema de distribución hacia la fuente para confirmar su integridad física, la capacidad que persiste y la calidad bacteriana y química del agua suministrada; se debe garantizar su cloración y hacer el tratamiento correspondiente. Se recomienda aumentar los niveles de cloro residual y elevar la presión del sistema de distribución del agua como parte de las operaciones de socorro. La baja presión aumenta la probabilidad de que se filtren agentes contaminantes en las tuberías maestras. ${ }^{10}$

En situaciones de emergencia se recomienda un nivel mínimo de cloro de 0,7 mg/L. Los análisis rutinarios del cloro residual deben comenzarse de inmediato, si no se dispone de instrumentos, se comprobará que el agua huele a cloro. Si las pruebas indican ausencia de cloro residual en el agua potable, se considerará probable la contaminación bacteriana, salvo que los análisis bacteriológicos demuestren lo contrario, y estos análisis necesitan un largo período de incubación (8-24 h), mientras que el cloro residual puede medirse en el terreno en pocos minutos. ${ }^{9}$

Si se sospecha que la fuente de agua está contaminada, se deben buscar fuentes alternativas de agua: acuíferos profundos y aguas de manantiales, agua de lluvia o aguas superficiales.

La falta de higiene es la principal causa de las enfermedades transmitidas por alimentos durante un desastre. Cuando se usan programas de alimentación en campamentos, el saneamiento de las cocinas es de gran importancia. Los utensilios deben lavarse con agua hervida o tratada y debe controlarse la higiene personal de los que preparan los alimentos.

Las provisiones de alimentos deben almacenarse en recipientes que eviten la contaminación por roedores e insectos.

Muchas enfermedades transmisibles se propagan a través de la contaminación fecal del agua y los alimentos, y por esto, hay que hacer una adecuada eliminación de excretas. ${ }^{2,11-13}$

Es posible que durante o después de una situación de emergencia, los alimentos no estén en buen estado para consumirlos. Es necesario identificar y eliminar los alimentos cuyo consumo pudiera ser peligroso. ${ }^{2,13}$

Es necesario enviar a la basura cualquier alimento que haya podido estar en contacto con el agua de una tormenta o inundación, botar los alimentos que tengan olor, color o texturas anormales; eliminar los alimentos perecederos (incluidos la carne, el pollo, el pescado, los huevos y las sobras) que hayan estado a $35^{\circ} \mathrm{C}$ o 
más, por $2 \mathrm{~h}$ o más; los alimentos descongelados que tengan cristales de hielo o que hayan estado a menos de $4,5^{\circ} \mathrm{C}$ pueden cocinarse $o$ volverse a congelar, botar todos los alimentos enlatados cuyos envases estén abiertos, dañados o inflados; los recipientes de alimentos con tapas que se abren manualmente y los alimentos enlatados se deben desechar si han estado en contacto con las aguas de la inundación ya que no se pueden desinfectar. Si las latas han estado en contacto con el agua de una tormenta o inundación, retirar las etiquetas, lavarlas y meterlas en una solución de 1 taza de cloro de uso doméstico con 5 galones $(19$ L) de agua. Identificar las latas nuevamente. ${ }^{8}$

\section{PROGRAMA DE ALIMENTACIÓN Y NUTRICIÓN}

Existe el Programa de Alimentación y Nutrición como parte del Plan Nacional de Emergencia en Cuba.

Se puede obtener la información sobre la disponibilidad de alimentos y la existencia de alimentos utilizables, a partir de un estimado inicial de las posibles necesidades de alimentos en la zona, de forma que puedan darse los pasos necesarios para conseguirlos, transportarlos, almacenarlos y distribuirlos; información sobre estructura y dinamismo del consumo alimentario, registros e inventarios para programar reservas.

La evaluación de los alimentos disponibles en almacenes consiste en identificar la cantidad de alimentos existentes por tipos, y para qué tiempo (número de días) son suficientes estos alimentos.

Las estimaciones de las necesidades nutricionales se realizan con la información específica: la estructura demográfica de la población por grupos de edades, sexo y localización, disponibilidad de alimentos, grupos de riesgo como son los enfermos, embarazadas, madres que lactan, niños (menores de 1 año, 1-5 años, 5-10 años), porcentaje de mujeres, ancianos y discapacitados, tasa de mortalidad infantil y causas de muerte (principales causas de muerte general), tasas de enfermedades de transmisión digestiva (EDA), otras y la situación nutricional de la población. Las necesidades aumentarán si la población está desnutrida, en niños, embarazadas y adultos, así como el índice de bajo peso al nacer.

La alimentación de emergencia consiste en el suministro de alimentos a personas sin acceso a estos por catástrofe natural, por tanto tiempo como sea necesario hasta su alimentación normal, sobre todo a poblaciones aisladas, instituciones y personal de ayuda.

\section{Cálculo de una dieta de emergencias ${ }^{14}$}

Se hace a partir de la cantidad energética total de la dieta.

Una dieta de supervivencia debe tener una cantidad total de energía de 1800 $\mathrm{kcal} / \mathrm{dí}^{1}{ }^{15}$

También existen recomendaciones de energía para situaciones de emergencias. ${ }^{15}$

Recomendaciones de energía en situaciones de desastre tabla 1. 
Tabla 1. Recomendaciones de energía en situaciones de desastres

\begin{tabular}{|l|c|c|}
\hline $\begin{array}{l}\text { Edad, vulnerables } \\
\text { y sexo }\end{array}$ & $\begin{array}{c}\text { Subsistencia } \\
\text { en emergencia (Kcal.) }\end{array}$ & $\begin{array}{c}\text { Mantenimiento temporal } \\
\text { (Kcal.) }\end{array}$ \\
\hline 0 a 2 años & 1000 & 1000 \\
\hline 3 a 5 años & 1250 & 1500 \\
\hline 6 a 9 años & 1500 & 1750 \\
\hline 10 a 17 años & 2000 & 2500 \\
\hline $\begin{array}{l}\text { Embarazo } \\
\text { y lactancia }\end{array}$ & 2000 & 2500 \\
\hline & Adultos & \\
\hline Hombres & 1900 & 2200 \\
\hline Mujeres & 1600 & 1800 \\
\hline
\end{tabular}

La distribución porcentual energética (DPE) de una dieta en emergencias es de 5 a $10 \%$ de la energía en proteínas, el $30 \%$ en grasas, y el $65 \%$ en hidratos de carbono.

Al inicio el alimento debe aplacar el hambre y sostener la moral, más que seguir los patrones nutricionales rígidos. El objetivo primario es sostener la vida en el transcurso de los días.

Los requerimientos nutricionales para dos semanas o menos, son cuantitativos y relativamente en menor cantidad que para los periodos largos. Esenciales para periodos cortos son el agua y los alimentos energéticos. ${ }^{5}$

\section{Forma práctica de elaborar una dieta en una situación de emergencia}

Cuatro toneladas de víveres por día servirán para alimentar a unas 10000 personas. Además de los productos básicos, se necesita aceite de cocina, verduras, sal, un alimento suplementario (puede ser leche en polvo, carne en conserva, pescado) y también son útiles desde el punto de vista psicológico, aunque no indispensables, té, café, azúcar y especies. Si no pueden obtenerse otros productos, la distribución de un único cereal, raíces o tubérculos serán suficientes para cubrir las necesidades nutritivas básicas, lo mejor será distribuir alimentos cocinados, por ejemplo, arroz hervido o pan.

No es necesario hacer un cálculo detallado del contenido vitamínico, mineral o proteínico de los alimentos distribuidos en la fase inicial, aunque sí deben ser aceptables y apetitosos, lo más importante es que proporcionen la energía adecuada. ${ }^{15}$

\section{Planificación de energía y nutrimentos según las necesidades medias de la población en la fase inicial de una emergencia $13,15,16$}

La cantidad total de calorías de una dieta de una persona adulta promedio oscila entre 2400 y $2800 \mathrm{Kcal}$. 
En la tabla 2 se aprecia las necesidades medias de la población por nutriente.

Tabla 2. Planificación de nutrientes y energía en el inicio de una emergencia

\begin{tabular}{|c|c|}
\hline Nutriente & Necesidades medias de la población \\
\hline Energía & $2100 \mathrm{Kcal}$. \\
\hline Proteínas & $10 \%-12 \%$ de la energía total $(52-63 \mathrm{~g}),<15 \%$ \\
\hline Lípidos & $17 \%$ de la energía total $(40 \mathrm{~g})$ \\
\hline Vitamina $\mathrm{A}$ & $1666 \mathrm{UI}^{*}$ (0 0,5 mg de equivalentes de retinol) \\
\hline Tiamina $\left(B_{1}\right)$ & $0,9 \mathrm{mg}$ (o 0,4 mg por ingesta de1 $000 \mathrm{Kcal}$ ) \\
\hline Riboflavina $\left(\mathrm{B}_{2}\right)$ & $1,4 \mathrm{mg}$ (0 0,6 mg por ingesta de $1000 \mathrm{Kcal}$. ) \\
\hline Niacina $\left(B_{3}\right)$ & $12,0 \mathrm{mg}$ (ó 6,6 mg por ingesta de $1000 \mathrm{Kcal}$. ) \\
\hline Vitamina C & $28,0 \mathrm{mg}$ \\
\hline Vitamina D & $3,2-3,8 \mu \mathrm{g}$ de calciferol \\
\hline Hierro & $22 \mathrm{mg}$ (baja biodisponibilidad, es decir, 5-9 \%) \\
\hline Yodo & $150 \mu g$ \\
\hline
\end{tabular}

Algunas de las raciones alimentarias suministradas pueden no ser suficientes para satisfacer las necesidades nutricionales (particularmente de riboflavina, niacina, vitamina $\mathrm{C}$, hierro y ácido fólico). Cuando en la evaluación se indica que la propia población no podrá satisfacer las necesidades de ciertos micronutrientes, en la intervención se deben prever medidas para subsanar esa carencia. Las diferencias a que puede dar lugar la distribución de raciones o compartir la comida en el grupo familiar inciden en la cantidad de alimentos que consumen las personas. Las pérdidas de micronutrientes se pueden producir durante el transporte y el almacenamiento de los alimentos, durante la elaboración, la molienda, como resultado de una cocción prolongada, en el caso de las vitaminas solubles en agua o que como consecuencia de la combinación de nutrientes en la dieta que impiden su absorción en el intestino (los fitatos obstaculizan la absorción del hierro procedente de fuentes vegetales). Las pérdidas también pueden producirse como consecuencia de una enfermedad, en particular las parasitarias y cuando la capacidad del organismo para obtener y asimilar los nutrientes está restringida.

Para prevenir las deficiencias de micronutrientes es necesario aumentar la cantidad de alimentos de la ración general para que pueda haber un mayor intercambio de alimentos, mejorar la calidad nutricional de la ración, abastecer de productos ricos en micronutrientes como suplemento de las raciones, enriquecer apropiadamente los alimentos básicos o los alimentos combinados y la suplementación medicinal de la Organización Mundial de la Salud. ${ }^{17,18}$

La anemia ferropénica, particularmente en mujeres embarazadas y adolescentes en crecimiento, tiene una incidencia importante en la morbilidad. La prevalencia de esta carencia puede haber aumentado o no a raíz de la emergencia. ${ }^{18}$

Posteriormente se debe determinar el porcentaje de personas con desnutrición energética nutrimental, la cantidad de personas fallecidas y enfermas y en qué medida se satisfacen sus necesidades nutricionales. Se debe realizar interrogatorio a los casos afectados por el desastre, de cómo es la alimentación y detectar insuficiencias. 
Para determinar las personas desnutridas se buscan los signos y síntomas de esa afección. Se puede medir la circunferencia braquial y los pliegues cutáneos.

En los niños se hará la toma del peso, la talla y la circunferencia del brazo (CB). Si es posible se puede recoger la fecha de nacimiento para calcular la edad exacta y la edad decimal. La desnutrición más grave es consecuencia de la exacerbación aguda de una desnutrición crónica. ${ }^{18}$

En situaciones de emergencia, el peso en relación con la talla es el mejor indicador de cambios agudos en el estado de nutrición. Los puntos de corte antropométricos en niños (para el porcentaje de la mediana): peso/talla, menos del $80 \%$ indica emaciación o desnutrición; peso/edad, menos del $80 \%$, falla de peso. La talla/edad, cuando es menos del $90 \%$, enanismo.

Para los percentiles: percentil peso/talla, si es < 3er. percentil, desnutrición; peso/ edad si es $<3$ es desnutrición, y baja talla si la talla para la edad es $<$ 3er.percentil. ${ }^{15,18}$

La circunferencia braquial (CB) de niños bien nutridos y en las edades de 1-5 años, es $>14 \mathrm{~cm}$. Una CB baja indica bajo peso. $L a C B>13,5 \mathrm{~cm}$, indica un niño no desnutrido, entre 13,5-12,5 cm, desnutrición moderada y $<12,5 \mathrm{~cm}$, desnutrición grave. ${ }^{18}$

En la evaluación antropométrica en adultos se utiliza el Índice de Masa Corporal (IMC) que se calcula: kg/talla ${ }^{2}$. El peso en $\mathrm{kg}$ y la talla en metros. El IMC: $<16 \mathrm{~kg} /$ $\mathrm{m}^{2}$ indica desnutrición energético nutrimental grave; de 16,0-16.9, desnutrición moderada; de $17,0-18,4 \mathrm{~kg} / \mathrm{m}^{2}$, leve y normal si el IMC está entre $18,5-24,9 \mathrm{~kg} /$ $\mathrm{m}^{2} .18$

En las embarazadas la evaluación consta de 3 aspectos: evaluación del peso al inicio del embarazo, evaluación de la circunferencia del brazo y la evaluación del incremento de peso durante el embarazo. La clasificación del IMC al inicio del embarazo: < 18,8 kg / $\mathrm{m}^{2}$ bajo peso; de 18,8 a $25,4 \mathrm{~kg} / \mathrm{m}^{2}$ peso norma; sobrepeso de 25,5 a 28,5 y $>28,6 \mathrm{~kg} / \mathrm{m}^{2}$, obesas. ${ }^{19}$

La circunferencia del brazo cuando es menor de 23,5 cm: desnutrición, y $<20,4$ $\mathrm{cm}$, desnutrición grave. La evaluación del incremento de peso según peso inicial: la embarazada que comienza su gestación con bajo peso, debe aumentar de 12,5 a $18,0 \mathrm{~kg}$, la normopeso de 11,5 a $16,0 \mathrm{~kg}$, el sobrepeso de 7,0 a $11,5 \mathrm{~kg}$ y las obesas, $6,0 \mathrm{~kg} \cdot{ }^{19}$

En Cuba está implementado el Sistema de Vigilancia Alimentaria y Nutricional (SISVAN) y un sistema de alerta de inseguridad alimentaria, que ofrece indicadores no solo del estado nutricional, sino también elementos acerca de la accesibilidad y el consumo de alimentos. ${ }^{20}$

Los desastres naturales azotan a Cuba y se deben atenuar sus estragos sobre la salud de la población, mediante la prevención oportuna para lograr la rehabilitación y reconstrucción en las áreas afectadas. Se debe mejorar el abastecimiento de alimentos con un mayor aprovechamiento de los productos cosechados, ampliar la cultura nutricional, con lo que se elevarían los niveles de la seguridad alimentaria y se prevendría la desnutrición.

\section{REFERENCIAS BIBLIOGRÁFICAS}


1. Organización Panamericana de la Salud. Los desastres naturales y la protección de la salud. Washington: OPS;2000. [Publicación científica No.675].

2. Los desastres naturales. Atención Primaria de Salud. Efectos generales de los desastres sobre la Salud. Villa Centenario OPS: Vivienda y comunidad saludable OPS [sitio en Internet]. 2003 [citado 2009]. Disponible en:

http://www.bvsde.paho.org/bvsasv/e/fulltext/villa/villa.pdf

3. Estrategia Internacional para la Reducción de los Desastres (EIRD). Conferencia Hemisférica sobre Reducción de los Desastres. San José, Costa Rica [sitio en Internet]. 2009 [citado 2010]. Disponible en: http://www.eird.org

4. Instituto de Planificación Física. Programa Mundial de Alimentos. Análisis y cartografía de la Vulnerabilidad y la Inseguridad Alimentaria en Cuba. La Habana: Instituto de Planificación Física;2001.

5. Organización Panamericana de la Salud. Normas de la OPS. La Seguridad Alimentaria y Nutricional en Situaciones de Emergencia. OPS 2009 [sitio en Internet]. 2009 [citado 2010]. Disponible en:

http://www.paho.org/spanish/dd/ped/te nutresp.htm

6. Terry B, Rodríguez A. Una estrategia de atención primaria de salud en situaciones de desastres. Gac Sanit. 2005;19(1):76-9.

7. Centro para el Control y Prevención de las Enfermedades. Alimentos y agua después de un desastre natural [sitio en Internet]. 2008 [citado 2009]. Disponible en: http://www.redcross.org/preparedness/cdc spanish/FoodWater.asp

8. Programa Mundial de Alimentos. Manual sobre las evaluaciones de la Seguridad Alimentaria en emergencias, primera edición. Roma: UNDRO;2005.

9. Biotecnología, alimentos derivados de organismos genéticamente modificados transgénico. Nutrición en emergencias [sitio en Internet]. 2003[citado 9 May 2005]. Disponible en: http://www.nutrinet.org

10. Organización Panamericana de la Salud. Emergencias y desastres en sistemas de agua potable y saneamiento. Guía para una respuesta eficaz. Washington: OPS/OMS; 2001.

11. Vigilancia Epidemiológica sanitaria en situaciones de desastres. Guía para el nivel local. serie Manuales y Guías sobre desastres. No. 2.OPS-OMS. sep. 2000. [sitio en Internet]. 2000[citado 2009]. Disponible en: http://www.col.opsoms.org/desastres/VigilanciaGuiaAll.pdf

12. Díaz Lorenzo T, Caballero A, Valdes-Dapena M. Clinical epidemiological research at home of children with diseases trasmited by food. J Pediatric Gastroenterol Nutr. 2003:39(1):205-6.

13. Programa Mundial de Alimentos. Asociación Alternativa ¿Cómo Contribuir a la Alimentación de Personas Afectadas por los Desastres Naturales? Perú: Asociación Alternativa; 2007.

14. Centro Latinoamericano y del Caribe de Información en Ciencias de La Salud (BIREME). Alimentación de emergencia [sitio en Internet]. 19 Mar 2008 [citado 
2009]. Disponible en:

http://vcd.crid.or.cr/vcd/index.php/ALIMENTACI\%C3\%93N DE EMERGENCIA

15. La Seguridad Alimentaria y nutricional en situaciones de Emergencia. Manejo de los Alimentos en situaciones de Emergencia. Organización Panamericana de la Salud. Técnicas sobre desastres [sitio en Internet]. 2005 [citado 2009]. Disponible en: http://www.paho.org/spanish/dd/ped/te nutresp.htm

16. Plasencia D. Nutrición y alimentación en estado de emergencia. La Habana: INHA;2001.

17. Organización Mundial de la Salud. Vitamin A Supplements: a Guide to their use in the treatment and prevention of Vitamin A deficiency and xerophthalmia. 2da ed. Washington:OMS; 2000.

18. Organización Mundial de la Salud. The Management of Nutrition in Major. Geneva:OMS; 2000.

19. Díaz ME, Montero $M$, Jiménez $S$, Wong I, Moreno $V$. Diseño y confección de las tablas antropométricas de la embarazada cubana. La Habana: INHA,UNICEF; 2008.

20. Jiménez Acosta S. Métodos de medición de la seguridad alimentaria. Rev Aliment Nutr. 1995;9(1):1-2.

21. Díaz Lorenzo T, Caballero A, Valdes-Dapena M. Clinical epidemiological research at home of children with diseases trasmited by food. J Pediatric Gastroenterol Nutr. 2003:39(1):205-6.

Recibido: 9 de septiembre de 2009.

Aprobado: 4 de febrero de 2010.

María Matilde Socarrás Suárez. Calle H No. 354 e/ 15 y 17. Plaza, El Vedado 10400. La Habana, Cuba

Teléf.: 831 4181. E-mail: m.socarras@infomed.sld.cu, bolet@infomed.sld.cu 